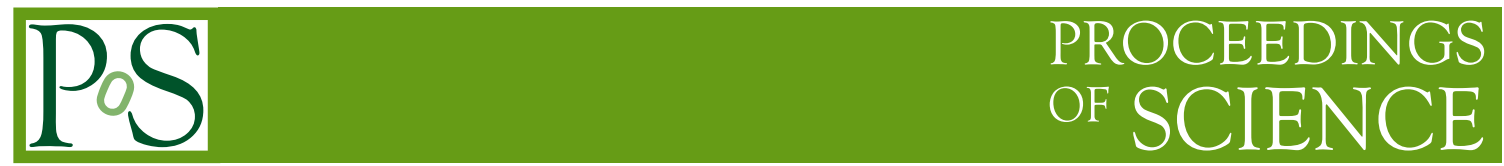

\title{
String Theory: Basic Facts and Recent Developments
}

\author{
Ashoke Sen* \\ Harish-Chandra Research Institute \\ Chhatnag Road, Jhusi, Allahabad 211019, India \\ E-mail: sen@hri.res.in, ashokesen1999@gmail.com
}

We review the basic facts and recent developments in string theory

35th International Conference of High Energy Physics - ICHEP2010,

July 22-28, 2010

Paris France

${ }^{*}$ Speaker. 


\section{String theory: basic facts}

In this section we shall review some of the basic facts of string theory.

1. Fundamental constituents of string theory are strings - one dimensional objects. They can be closed without any ends or open with two ends. The different vibrational states of the string will correspond to different elementary particles in the theory.

2. String theory is formulated consistent with the principles of

- Quantum mechanics and

- Special theory of relativity.

3. One of the vibrational states of the closed string has the properties expected of a graviton, the mediator of gravitational force. Thus string theory automatically contains gravity.

4. The requirement of satisfying the laws of quantum mechanics and special theory of relativity puts strong constraints on the theory:

- String theory is consistent in $9+1$ dimensional space-time instead of the usual $3+1$ dimensional space-time.

- In (9+1) dimensions there are only five fully consistent string theories, known as type IIA, type IIB, type I, $S O(32)$ heterotic and $E_{8} \times E_{8}$ heterotic string theories.

5. Often consistency demands that a string theory contains objects other than the fundamental strings, $e . g$. $p$-dimensional extended objects known as D- $p$-branes.

6. The problem of having six extra dimensions can be resolved using the idea of compactification. The main idea behind compactificaion is to take 6 of the 9 dimensions to describe a small compact space instead of infinite flat space. When the size of the compact space is smaller that the resolution of the most powerful microscope, the space will appear to be 3 dimensional.

7. There are many possible ways to compactify string theory. These different compactifications correspond to different phases / vacua of string theory. The apparent laws of nature e.g. spectrum and interactions of the elementary 'particles' depends on which phase of string theory we are in. Some phases of string theory come quite close to the theory that describes our world. For example, besides gravity they also contain gauge interaction, chiral fermions etc.

8. String theory exhibits duality symmetries, implying that often a particular phase of string theory can be viewed in more that one ways, - as different compactifications of different string theories. Schematically we can view string theory like a big room with five windows, with different objects in the room representing different phases and the five windows representing the five string theories. This picture of a universal theory is often known as the M-theory. 
9. For more than 30 years it has been known that black holes carry non-zero entropy given by

$$
A / 4 G_{N}
$$

where $A$ is the area of the event horizon, $G_{N}$ is the Newton's constant, and we have used units in which $\hbar=1, c=1$ and $k_{B}=1$. Given this, it is natural to ask: can we find a statistical interpretation of this entropy as $\ln \Omega$ where $\Omega$ is the number of quantum states of the system? 15 years ago Strominger and Vafa answered this in the affirmative for extremal black holes carrying large charges. Here $\Omega$ is computed simply by counting all states with a given charge and mass, without any reference to whether they correspond to horizon or interior degrees of freedom. This makes the agreement all the more remarkable since it relates the result of a counting problem to a purely geometrical quantity.

10. One of the most exciting duality symmetries of string theory is the AdS/CFT correspondence - an observation that some phases of string theory actually have dual descriptions as conventional field theories without gravity. According to this correspondence, string theory on $A d S$ space $\times$ a compact space $K$ with metric

$$
d s^{2}=\frac{d z^{2}+\eta_{\mu \nu} d x^{\mu} d x^{\nu}}{z^{2}}+d s_{K}^{2}
$$

is dual to a conformally invariant quantum field theory living at the boundary $z=0$.

11. The main obstacle to connecting string theory to experiment is the existence of huge number of phases of string theory. This is often refered to as the string landscape. It is even conceivable that different parts of the universe are in different phases of string theory, and we happen to see one particular phase because we live in some particular region of the universe.

12. The existence of huge number of phases of string theory has been used to propose a tentative resolution of the fine tuning problem. Fine tuning problem stems from the fact that some observed parameters of the universe, like the cosmological constant, are much smaller that their natural values which we get from dimensional argument. Thus requires a delicate adjustment of parameters. However given the existence of huge number of phases of the theory, it is conceivable that in some of these phases such cancelations will occur accidentally. This still leaves open the question as to why we live in such a phase, but one can argue that the present form of life is possible only in a universe where such delicate cancelations occur.

The existence of the landscape in string theory implies that unless we can identify precisely which phase describes the environment in which we live, we cannot make precise predictions which can be tested in experiment.This is still an unresolved problem. Given this situation it would seem that until we can find the right phase of string theory which describes our world, there cannot be any further progress. Despite this there has been significant progress in various directions, often by cleverly turning the landscape to our advantage. In the rest of this talk I shall describe some of the recent developments in string theory, with some focus on the parallel session talks presented at this conference. For appropriate references to these talks I refer the reader to the ICHEP web-site ichep 


\section{Recent Developments}

Recent developments in string theory can be divided roughly into five classes although the boundary between them is not a sharp one.

1. Conceptual issues

2. Developments within string theory

3. Exploring the landscape

4. Applied string theory

5. String motivated / induced developments in quantum field theory

I shall try to present few examples of each type.

\subsection{Conceptual issues:}

Although different points in the string landscape describe very different physics, various theoretical issues, in particular those in quantum gravity, are common to either all or a wide variety of phases of string theory. As a result these issues can be addressed without knowing which particular phase of string theory describes our world. Two of the talks at ICHEP fall into this category:

1. Gravity as an effective theory.

2. Black hole thermodynamics

\subsubsection{Gravity as an effective theory}

In string theory it has been known for a while that the gravitational degrees of freedom can emerge from another structure where gravity does not appear as fundamental degree of freedom. The oldest example of this is the duality between open and closed strings. Since gravitons arise as vibrational state of a closed string, a closed string theory can be regarded as one where gravity is fundamental. On the other hand vibrational states of an open string do not contain any graviton. Nevertheless one finds that closed string appear as intermediate states in the scattering amplitude of open strings at the loop level. Thus in a sense closed strings, including gravity, emerge in a quantum theory of open strings. This takes a much more dramatic form in AdS/CFT correspondence, where gravity propagating in the bulk of AdS emerges from an ordinary gauge theory living on the boundary of Ads space.

The proposal by Verlinde[1], presented in this conference, is in the same spirit, but goes beyond. Here the gravitational force is regarded as the 'entropic force' - force obtained as the result of a system trying to maximize its entropy. Thus gravity emerges from the statistical mechanics of some other fundamental degrees of freedom in the large volume limit. It is perhaps fair to say that this proposal is still in its infancy, and a concrete model, based on string theory or otherwise, of the microscopic degrees of freedom is needed to make this into a concrete proposal. This will also clarify in what way this differs from the already existing understanding of emergent gravity in string theory. 


\subsubsection{Black hole thermodynamics}

Earlier we had discussed the success of string theory in providing a microscopic derivation of black hole entropy. However the agreement between $\ln \Omega$ and $S_{B H}$ is not exact, - it is valid only in the limit of large charges, when the number of quantum states is large. From this one may be tempted to conclude that the microscopic description is the correct description, and geometry is an 'emergent phenomenon', 1.e. an approximate description of the system when there are large number of quantum states. More generally we may be tempted to conclude that gravity, or the theory of closed strings which contains it, is also an emergent phenomenon.

However duality symmetries have shown us that an 'emergent phenomenon' in one description may be part of the fundamental description in a dual formulation. Thus whether a phenomemon is emergent or not may depend on the description we are using. Could the situation with black holes be similar, 1.e. can it be that the description using gravity / closed strings can also fully describe the system? To test this we need to test if the description using geometry / gravity / closed strings can reproduce the exact result for the number of microstates, instead of just the leading behaviour for large charges. This requires us to study corrections to the 'area formula' for the black hole entropy by taking into account

1. Stringy corrections

2. Quantum corrections

At the same time the gravity description should also be able to calculate the other properties of black holes, e.g. whether the states represented by black hole are bosons or fermions, what is the distribution of quantum numbers (other than charges) among the quantum states etc. As described by me in the parallel session talk, $A d S_{2} / C F T_{1}$ correspondence, - a version of AdS/CFT correspondence - now provides us with a specific algorithm to compute all these quantities in the gravity description, and many tests of this correspondence has been carried out[2].

Another aspect of black hole thermodynamics that has been discussed in this conference by El-Shawk is whether we can use classical solutions in gravity to understand the black hole microstates[3, 4]. This approach, known as the fuzzball proposal, has been investigated intensely in recent years.

\subsection{Developments within string theory}

The goal here is to find new results in string theory which enhances our knowledge and understanding of the theory. I shall describe several such examples below.

\subsubsection{Theory of membranes in 11 dimensional supergravity}

One of the phases of string theory has 11 dimensional supergravity as its low energy limit. This theory is known to have classical membrane solutions. The low energy dynamics of a single membrane is described by a supersymmetric $\mathrm{U}(1)$ gauge theory. One of the open problems which had been around for several years was to find the theory that describes the low energy dynamics of several coincident membranes. This problem was finally solved due to the work of Bagger and Lambert[5, 6, 7], Gustavson[8] and finally Aharony, Bergman, Jafferis, Maldacena[9]. We now have an understanding of the low energy dynamics of multiple membranes not only in flat 11 
dimensional space-time, but also for membranes in complicated background. This was reported in this conference by Sparks[10, 11].

These theories are also significant since they are dual to string theory on $A d S_{4}$ times a compact space, - close cousin of the four dimensional de Sitter space in which we live. From the study of supergravity in $A d S_{4}$ it was predicted that the dual theory of $N$ coincident membranes will have its partition function growing as $N^{3 / 2}$ for large $N$. This has now been verified[12].

\subsubsection{Exact results for higher derivative corrections}

Green, Miller, Russo, Vanhove[13] found some exact results for certain higher derivative corrections to the low energy effective action (including all loop and non-perturbative corrections). This work is significant since it shows the power of duality symmetries to constrain the effective action of string theory. Furthermore the ability to construct the effective action required the existence of certain functions with certain modular properties and stringent boundary conditons, and the fact that such functions exist provides us with an internal consistency test of string theory.

\subsubsection{Exact classical solutions in string theory}

Another development in this direction is the construction of exact classical solutions of string theory. This started with the work of Schnabl[14] several years ago where he gave an exact solution describing the tachyon vacuum of bosonic open string theory. In this conference he reported preliminary results on the construction of multiple D-brane solutions in the open string field theory living on a single D-brane.

\subsection{Exploring the landscape}

In absence of a general principle, identifying the precise phase of string theory in which we live seems to be a difficult problem. However it is still a fruitful exercise to see how close we can get to the real world. This could give us a clue to where and how to look for the real world in the landscape, and how our part of the universe could have arrived there by cosmic evolution.

Most well studied cases are F-theory models with flux. An general framework for constructing de Sitter phase of string theory was developed by Kachru, Kallosh, Linde and Trivedi[15]. Here they first construct type IIB compactification on $A d S_{4} \times$ Calabi-Yau and then lift $A d S_{4}$ to $d S_{4}$ by adding some supersymmetry breaking anti-branes. Halmagyi reported his recent results with Bena and Grana[16] in which they carry out a detailed study of the effect of adding the anti-brane. Their analysis indicates that the effect of the anti-brane may be more subtle than was previously thought. However more analysis is needed before one can draw a definite conclusion.

Many other studies of type IIB / F-theory compactification have been carried out during the last few years yielding models closer to the observed world. A popular direction of study is that of local F-theory models where one tries to examine local structure of 7-branes without worrying about how to eventually put this inside a Calabi-Yau manifold[17, 18]. This has the advantage of being able to focus on the aspect of F-theory that determines the low energy particle physics without gravity.

However it is also important to explore other possible ways of getting de Sitter vacua in string theory. In this conference Petrini reported her work[19] on possible construction of de Sitter vac- 
uum from compactification of type IIA string theory. At present it remains unclear if one can get a fully consistent de Sitter vacuum from this approach.

Another active area of research has been string cosmology, - in particular models of inflation in string theory. Most studied case has been inflation via brane - anti-brane annihilation, where the inflationary phase corresponds to slowly moving brane-antibrane system, separated along some compact directions, and the inflaton field is associated with the distance between the brane and the anti-brane. The end of inflation is signalled by brane-antibrane annihilation via tachyon condensation. The main issue here is how to get an extremely flat inflaton potential? There is still no completely natural mechanism, and all models of this type require some degree of fine tuning. A comprehensive recent study on this can be found in [20].

It is however important to also explore other aspects of string cosmology. Some different aspects of string cosmology was reported in this conference by Estes based on the work of [21].

\subsection{Applied string theory}

As we have already discussed, string theory landscape includes many phases which do not describe the universe we live in. However many of these phases may have phenomena similar to what we observe in our universe, and hence can be used to study these phenomena. Some classic examples of this approach are dynamics of strongly coupled gauge theories[22, 23] and study of superconductivity[24, 25].

Basic tool in these studies is AdS/CFT correspondence. We consider an appropriate gravitational background with asymptotic AdS geometry. By AdS/CFT correspondence this describes a conformal field theory living on the boundary of AdS. By choosing the gravitational background appropriately one can try to ensure that the CFT at the boundary describes the physics we are interested in. For example superconductivity requires a U(1) gauge symmetry that is unbroken near the boundary of $A d S$ space but is broken in the interior by non-vanishing background value of a charged field.

This has been an extremely active area of research during the last few years. I shall just list few of the results found using this approach.

1. For a wide variety of strongly coupled conformally invariant gauge theories with $A d S$ dual, the finite temperature low energy dynamics is controlled by hydrodynamic equations with universal coefficients[26].

2. Using dual $A d S$ description one can find examples of quantum systems in $2+1$ dimensions with non Fermi liquid behaviour[27].

Talks presented in the conference related to this approach are those by Niarchos, Wallon and Skenderis.

\subsection{String motivated developments in quantum field theory}

Recent years have seen many string inspired developments in quantum field theories. In one way these developments differ from the applied string theory discussed in the previous section. While the developments described below have been inspired by insights from string theory, in 
principle they could be carried out without invoking string theory. In contrast the developments described in the previous section make use of AdS/CFT correspondence in an essential way.

We list below some examples of recent developments in quantum field theories.

1. We now have exact results for anomalous dimensions of operators in $\mathscr{N}=4$ super YangMills theories in the planar limit. This began with the observation that the problem of finding the anomalous dimension of certain operators in $\mathscr{N}=4$ supersymmetric Yang-Mills theories can be mapped to the problem of finding the spectrum of an integrable spin chain[28]. Following many generalizations and extensions of these results, we now have a concrete proposal of the exact anomalous dimension of a class of operators in the planar limit[29].

2. In another line of development, many exact results for on-shell S-matrix elements in $\mathscr{N}=$ 4 supersymmetric Yang-Mills theories have been derived using on-shell methods. These include in particular all tree amplitudes. Furthermore we also have many constraints on loop amplitudes which might eventually give a complete understanding of perturbative S-matrix elements in string theory. This work is also a result of concerted effort by many groups. A recent references on this subject is [30] where more references can be found.

3. In a remarkable series of papers Gaiotto and his collaborators developed tools to construct and study a whole new class of $\mathscr{N}=2$ superconformal field theories in four dimensions[31, 32] - some without even a Lagrangian description. Furthermore many exact results for low energy effective action, Wilson line expectation values etc. have been found for these theories.

4. Possible finiteness of $\mathscr{N}=8$ supergravity has been an active area of research during the last few years. The debate is on but the final verdict on this is still awaited.

I shall end this section by describing some of the talks in this conference which fall in the category of string inspired developments in quantum field theory.

1. Korchemsky reviewed the fact that tree level scattering amplitudes in $\mathscr{N}=4$ supersymmetric Yang-Mills theory have not only the usual conformal symmetry but also dual superconformal symmetry acting on momentum space[33]. This provides strong constraints on amplitudes.

2. Rychkov described how crossing symmetry of four dimensional conformal field theories can be used to put bounds on anomalous dimensions and operator product expansion coefficients[34, 35, 36]. These bounds are relevant for constraining technicolour and unparticle models.

3. Johansson described some simple rules for constructing gravity amplitudes from Yang-Mills amplitudes[37, 38].

4. Vanhove gave an overview of the relationship between gauge and gravity amplitudes[39, 40, $41,42]$ and described how the the rules described by Johansson can be used to argue that the cancellation of ultraviolet divergence of $\mathscr{N}=8$ supergravity will stop at 7 loops unless there are some 'accidental' cancelations. 
Besides the various string theory related topics described above, there were also two talks outside string theory. Barrau described loop quantum gravity and its application to cosmology for homogeneous universe, while Ward discussed the possibility of getting asymptotically safe gravity after resummation of Feynman graphs.

\section{Conclusion}

I hope I have convinced you that during the last few years string theory has made progress in diverse directions, both on the formal and the applied side. The history of the subject shows that progress in this subject come from unexpected directions, and often the development in any one direction leads to developments in many other directions. Thus it is important to keep an open mind and try to make progress in whatever direction one can.

\section{References}

[1] E. P. Verlinde, [arXiv:1001.0785 [hep-th]].

[2] I. Mandal, A. Sen, Class. Quant. Grav. 27, 214003 (2010). [arXiv:1008.3801 [hep-th]].

[3] J. de Boer, S. El-Showk, I. Messamah et al., JHEP 0905, 002 (2009). [arXiv:0807.4556 [hep-th]].

[4] J. de Boer, S. El-Showk, I. Messamah et al., JHEP 1002, 062 (2010). [arXiv:0906.0011 [hep-th]].

[5] J. Bagger, N. Lambert, Phys. Rev. D75, 045020 (2007). [hep-th/0611108].

[6] J. Bagger, N. Lambert, Phys. Rev. D77, 065008 (2008). [arXiv:0711.0955 [hep-th]].

[7] J. Bagger, N. Lambert, JHEP 0802, 105 (2008). [arXiv:0712.3738 [hep-th]].

[8] A. Gustavsson, Nucl. Phys. B811, 66-76 (2009). [arXiv:0709.1260 [hep-th]].

[9] O. Aharony, O. Bergman, D. L. Jafferis et al., JHEP 0810, 091 (2008). [arXiv:0806.1218 [hep-th]].

[10] N. Benishti, Y. -H. He, J. Sparks, JHEP 1001, 067 (2010). [arXiv:0909.4557 [hep-th]].

[11] N. Benishti, D. Rodriguez-Gomez, J. Sparks, JHEP 1007, 024 (2010). [arXiv:1004.2045 [hep-th]].

[12] N. Drukker, M. Marino, P. Putrov, [arXiv:1007.3837 [hep-th]].

[13] M. B. Green, S. D. Miller, J. G. Russo et al., [arXiv:1004.0163 [hep-th]].

[14] M. Schnabl, [arXiv:1004.4858 [hep-th]].

[15] S. Kachru, R. Kallosh, A. D. Linde et al., Phys. Rev. D68, 046005 (2003). [hep-th/0301240].

[16] I. Bena, M. Grana, N. Halmagyi, JHEP 1009, 087 (2010). [arXiv:0912.3519 [hep-th]].

[17] C. Beasley, J. J. Heckman, C. Vafa, JHEP 0901, 058 (2009). [arXiv:0802.3391 [hep-th]].

[18] C. Beasley, J. J. Heckman, C. Vafa, JHEP 0901, 059 (2009). [arXiv:0806.0102 [hep-th]].

[19] D. Andriot, E. Goi, R. Minasian et al., [arXiv:1003.3774 [hep-th]].

[20] D. Baumann, A. Dymarsky, S. Kachru et al., JHEP 1006, 072 (2010). [arXiv:1001.5028 [hep-th]].

[21] J. Estes, C. Kounnas, H. Partouche, [arXiv:1003.0471 [hep-th]].

[22] G. Policastro, D. T. Son, A. O. Starinets, Phys. Rev. Lett. 87, 081601 (2001). [hep-th/0104066]. 
[23] T. Sakai, S. Sugimoto, Prog. Theor. Phys. 113, 843-882 (2005). [hep-th/0412141].

[24] S. A. Hartnoll, C. P. Herzog, G. T. Horowitz, Phys. Rev. Lett. 101, 031601 (2008). [arXiv:0803.3295 [hep-th]].

[25] S. S. Gubser, Phys. Rev. Lett. 101, 191601 (2008). [arXiv:0803.3483 [hep-th]].

[26] S. Bhattacharyya, V. EHubeny, S. Minwalla et al., JHEP 0802, 045 (2008). [arXiv:0712.2456 [hep-th]].

[27] H. Liu, J. McGreevy, D. Vegh, [arXiv:0903.2477 [hep-th]].

[28] J. A. Minahan, K. Zarembo, JHEP 0303, 013 (2003). [hep-th/0212208].

[29] N. Gromov, V. Kazakov, P. Vieira, Phys. Rev. Lett. 104, 211601 (2010). [arXiv:0906.4240 [hep-th]].

[30] N. Arkani-Hamed, J. Bourjaily, F. Cachazo et al., JHEP 1101, 049 (2011). [arXiv:0912.4912 [hep-th]].

[31] D. Gaiotto, [arXiv:0904.2715 [hep-th]].

[32] L. F. Alday, D. Gaiotto, Y. Tachikawa, Lett. Math. Phys. 91, 167-197 (2010). [arXiv:0906.3219 [hep-th]].

[33] J. M. Drummond, J. Henn, G. P. Korchemsky et al., Nucl. Phys. B828, 317-374 (2010). [arXiv:0807.1095 [hep-th]].

[34] R. Rattazzi, V. S. Rychkov, E. Tonni et al., JHEP 0812, 031 (2008). [arXiv:0807.0004 [hep-th]].

[35] V. S. Rychkov, A. Vichi, Phys. Rev. D80, 045006 (2009). [arXiv:0905.2211 [hep-th]].

[36] F. Caracciolo, V. S. Rychkov, Phys. Rev. D81 (2010) 085037. [arXiv:0912.2726 [hep-th]].

[37] Z. Bern, J. J. M. Carrasco, H. Johansson, Phys. Rev. D78, 085011 (2008). [arXiv:0805.3993 [hep-ph]].

[38] Z. Bern, J. J. M. Carrasco, H. Johansson, Phys. Rev. Lett. 105, 061602 (2010). [arXiv:1004.0476 [hep-th]].

[39] N. E. J. Bjerrum-Bohr, P. H. Damgaard, P. Vanhove, Phys. Rev. Lett. 103, 161602 (2009). [arXiv:0907.1425 [hep-th]].

[40] N. Berkovits, M. B. Green, J. G. Russo, P. Vanhove, JHEP 0911, 063 (2009). [arXiv:0908.1923 [hep-th]].

[41] N. E. J. Bjerrum-Bohr, P. H. Damgaard, T. Sondergaard, P. Vanhove JHEP 1006, 003 (2010). [arXiv:1003.2403 [hep-th]].

[42] P. Vanhove, [arXiv:1004.1392 [hep-th]]. 